\title{
Effect of plastic deformation on elastic and plastic recovery in CP- Titanium
}

\author{
Saber Khayatzadeh $^{\mathrm{a}}{ }^{*}$, Salah Rahimi ${ }^{\mathrm{b}}$ and Paul Blackwell ${ }^{\mathrm{c}}$ \\ Advanced Forming Research Centre \\ University of Strathclyde \\ 85 Inchinnan Drive, Renfrew (PA4 9LJ), Glasgow, United Kingdom \\ asaber.khayatzadeh@strath.ac.uk, bsalah.rahimi@strath.ac.uk, cpaul.blackwell@strath.ac.uk
}

Keywords: Springback, Sheet Forming, Plastic Deformation, CP-Titanium

\begin{abstract}
The springback associated with cold deep drawing of sheet metals leads to undesired dimensional changes in the final products. This is often due to the heterogeneous plastic deformation in different areas of the intended geometry that creates various stress states throughout the part. The major objective of this study is to understand the interconnection between springback, level of plastic deformation, degradation of elastic modulus and strain recovery in a CP-Ti material. The mechanical properties of the sheet material and the dependency of mechanical properties on directionality are investigated by examining samples from three orientations of parallel to the rolling direction (RD), at $45^{\circ}$ to $\mathrm{RD}$ and perpendicular to $\mathrm{RD}$. The degradation of elastic modulus as a function of level of plastic deformation was explored for $0^{\circ}$ and $45^{\circ}$ samples by conducting multistep uniaxial loading-unloading in tension.

The experimental results showed that the mechanical properties vary for each direction, with the lowest elastic modulus along RD. A significant degradation was observed in elastic modulus (up to $50 \%$ reduction) with increased plastic deformation. This resulted in more strain relaxation compared to that associated with the initial elastic modulus. For stresses below $100 \mathrm{MPa}$, a nonlinear (plastic) recovery was observed, resulting in additional relaxation in the total strain upon load removal in each step of the interrupted tests. This plastic recovery behaviour is observed to be dependent on sample orientation. It is concluded that accurate prediction of springback during sheet metal forming, requires a material model which takes into accounts the directional degradation of elastic modulus and the plastic recovery as a function of plastic deformation.
\end{abstract}

\section{Introduction}

Springback is one of the major engineering problems associated with products manufactured through sheet metal forming processes. Springback often causes deviation of the manufactured component profiles from their targeted theoretical profiles. The springback also changes the status of strain and stress in the deformed material following tool (e.g. punch) removal [1- 3]. The springback is stated to be the strain recovery in the formed product following the forming process [4]. This is more pronounced during sheet metal forming compared to other methods of forming (i.e. bulk forming), owing to the small thickness of the material.

During the sheet forming process, a large level of plastic deformation, varying in different directions, is applied to the part, which changes the material's inherent properties, such as its elastic modulus $(E)$. Therefore, in order to predict the springback effects more precisely, it is necessary to gain a good understanding of such changes in material properties. The interrupted tensile test is a common method that has been utilized to achieve better understanding of the dependency of mechanical properties (e.g. elastic modulus) on plastic deformation [4- 6]. Several studies have been performed to explore the effect of different scales of plastic deformation introduced during cold forming on mechanical properties [3- 6]. Benito et al [5] studied the decrease in elastic modulus in pure iron deformed by drawing at room temperature where $E$ decreased with increasing 
plastic deformation (to around 5\%), and thereafter a slight increase in $E$ was observed. The observed change in $E$ is linked to the structure of dislocations that are developed with cold deformation [5, 7]. Luo and Ghosh [4] argued that following the plastic deformation in deepdrawing of quality special killed (DQSK) steel, the strain recovery is nonlinear and greater than the amount expected on the basis of linear elasticity. It was shown that higher levels of plastic deformation, significantly reduced $E$, though, increased Poisson's ratio [4]. In general, a reduction in $E$ occurs with inelasticity, however, the opposite is also observed for some materials such as pure iron where a slight increase in $E$ is observed with plastic deformation. Hence, it is necessary to study these changes for $\alpha$-Titanium, to predict springback more accurately during forming.

Establishing a relationship between springback and the appropriate material model is an important step. Therefore, performing the necessary experiments on Titanium and gaining a thorough understanding of mechanical properties in different directions and the influence of plastic deformation on them are crucial. In the present study, a number of experiments were performed and these changes, affecting the accuracy of the springback predictive models, are considered.

\section{Experimental procedure}

In the present study, an uncoated cold-rolled sheet and annealed of commercially pure titanium (CP-Ti Grade 2) with a thickness of $1.6 \mathrm{~mm}$ was used. The titanium was supplied by TIMET in a form of a sheet with $1.6 \mathrm{~mm} \times 1 \mathrm{~m} \times 2 \mathrm{~m}(\mathrm{~T} \times \mathrm{W} \times \mathrm{L})$ dimensions. This material has an $\alpha$-phase microstructure, which consists of $\approx 100 \%$ of hexagonal close-packed (HCP) alpha phase at room temperature.

The specimens for uniaxial tensile tests were cut from the as-received sheet using Electrical Discharge Machining (EDM), in three orientations at $0^{\circ}, 45^{\circ}$ and $90^{\circ}$ to the rolling direction (RD), to investigate the anisotropic behaviour. In order to study the consistency in the material's behaviour, six samples were manufactured for each direction. The samples were designed based on ASTM E8/EM8-11 [8]; a schematic sketch of the sample along with its dimensions is shown in Fig. 1.

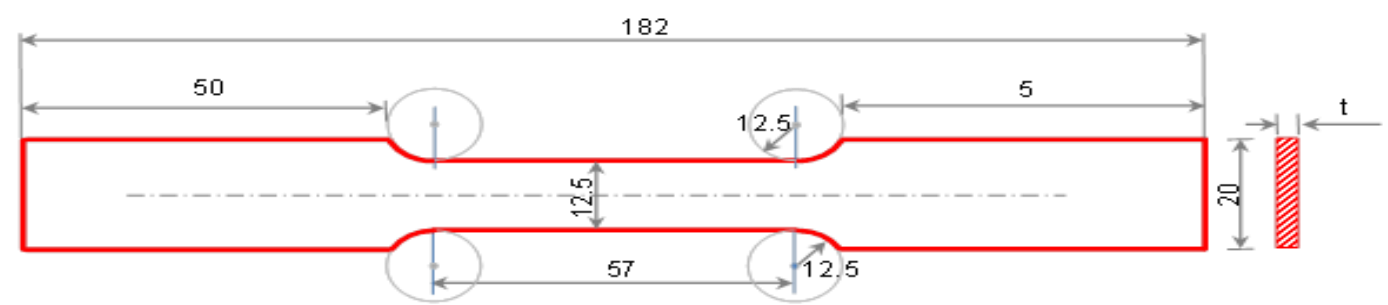

Fig. 1: Schematic illustration of a sample for uniaxial tensile test (ASTM E8/E8M) (All dimensions are in $\mathrm{mm}$ )

Standard Tensile Test. Uniaxial tensile tests were conducted using a Zwick Roell machine, to examine the mechanical properties of the material in different directions. The standard tensile tests were carried with two different strain rates of 0.01 and $0.002 \mathrm{~s}^{-1}$, one sample for each strain rate and two for each direction $\left(0^{\circ}, 45^{\circ}\right.$ and $\left.90^{\circ}\right)$. An external MTS type extensometer, capable of measuring strain in two directions ( $\mathrm{L}$ and $\mathrm{W}$ ), was employed to measure the changes in dimensions along and across the gauge length of the samples during testing.

Interrupted Tensile Test. A set of loading-unloading tests were conducted on the standard tensile samples (Fig. 1). These tests were conducted on samples at $0^{\circ}$ and $45^{\circ}$ to RD. The interrupted tests were conducted on the same machine used for the monotonic tests. The interrupted tensile tests were conducted using displacement control in which a number of deformation steps (i.e. loading and unloading) were applied. In each deformation step, on average 0.01 true plastic strain was achieved (at early stages of test). The tests were conducted with a strain rate of $0.01 \mathrm{~s}^{-1}$. The surface strain along and across the gauge length for each sample was mapped during the test using 3D Digital Image Correlation (DIC) technique, which is capable of mapping the displacement in-plane 
and out of plane during loading-unloading process, with a high resolution. Images of the sample surface were recorded using LaVision's MX4M digital cameras installed on a Zeiss Discovery.V12 3D stereo microscope. The time interval between observations was 0.1 seconds through the tests. The images obtained during the tests were analysed by the LAVision DaVis Image Correlation software (version 8.3.0) to obtain 3D strain maps. The strain maps were obtained using a $60 \times 60$ pixel window with $30 \%$ overlap. In these tests one pixel is equivalent to $39 \mu \mathrm{m}$. Displacements were therefore measured on the surface at positions with grid spacing of $1.6 \mathrm{~mm}$ in all cases.

\section{Results}

Table 1 summarises the results of the standard tensile tests conducted on the samples taken from three directions of the as-received material. For each test, elastic modulus $(E)$, failure strain $\left(\varepsilon_{f}\right)$, $0.2 \%$ of yield stress $\left(\sigma_{0.2}\right)$, and ultimate tensile stress $\left(\sigma_{U T S}\right)$ are provided. The elastic modulus is found to vary for different orientations of the sheet. An increase of over $10 \%$ is observed by changing the orientation from $0^{\circ}$ to $90^{\circ}$. The failure strain is higher for the samples oriented $45^{\circ}$ to the RD, compared to those of $0^{\circ}$ and $90^{\circ}$, and this was consistent for different strain rates. The yield point was also observed to change significantly with the sample orientation such that it was measured at $309 \mathrm{MPa}$ (for a strain rate of $0.01 \mathrm{~s}^{-1}$ ) for $0^{\circ}$, as opposed to $415 \mathrm{MPa}$ for $90^{\circ}$. However, the ultimate tensile strength showed a reversed order of behaviour as compared to that of the elastic modulus and yield point, where it was maximum at $0^{\circ}$ and $90^{\circ}$, and a minimum at $45^{\circ}$ orientation.

Fig. 2a shows the full engineering stress-strain curves for all samples as a function of orientation and strain rate. It is apparent that the work hardening is reduced by changing the orientation of the sample from $0^{\circ}$ to $90^{\circ}$ with respect to RD. Fig. $2 \mathrm{~b}$ highlights the early stages of the standard tensile tests (i.e. elastic region and early stage of work-hardening). A second yield point can be seen for samples at both $45^{\circ}$ and $90^{\circ}$, though it is more pronounced for the latter (Fig. 2).

Fig. 3 shows the true stress-strain curve of continuous (standard) and interrupted tensile tests for samples aligned with RD (i.e. $0^{\circ}$ orientation). This figure is an example of the interrupted tests with a strain rate of $0.01 \mathrm{~s}-1$. True stress and true strain were computed from engineering data set, assuming that the sample's volume remains constant after deformation in tension. As shown, the material during the loading-unloading process shows a similar behavior to that of the standard tensile test throughout the experiment. For each interrupted tensile test, two steps of loadingunloading were considered in the elastic region at stress levels of $80 \mathrm{MPa}$ and $200 \mathrm{MPa}$, before yield. In fact, the slope of the loading curve (i.e. elastic modulus) decreases with an increase in the level of plastic deformation after yielding. This is more evident in Fig. 4.

Table 1: Details of mechanical properties obtained from tensile tests conducted on the samples extracted in different directions.

\begin{tabular}{|c|c|c|c|c|c|c|}
\hline $\begin{array}{c}\text { Sample Orientation with respect } \\
\text { to RD }\end{array}$ & \multicolumn{2}{|c|}{$0^{\circ}$} & \multicolumn{2}{c|}{$\mathbf{4 5}^{\circ}$} & \multicolumn{2}{c|}{$90^{\circ}$} \\
\hline Strain Rate $\left(\mathrm{s}^{-1}\right)$ & $\mathbf{0 . 0 1}$ & $\mathbf{0 . 0 0 2}$ & $\mathbf{0 . 0 1}$ & $\mathbf{0 . 0 0 2}$ & $\mathbf{0 . 0 1}$ & $\mathbf{0 . 0 0 2}$ \\
\hline $\mathrm{E}(\mathrm{GPa})$ & 104.7 & 99.2 & 110.3 & 109.7 & 119.1 & 111.4 \\
\hline Failure Strain, $\left(\varepsilon_{f}\right)(\%)$ & 27.9 & 32 & 29.7 & 35 & 26.7 & 31 \\
\hline 0.2\% Yield $(\mathrm{Mpa}),\left(\sigma_{0.2}\right)$ & 309 & 301 & 367 & 342 & 418 & 387 \\
\hline Ultimate stress $(\mathrm{MPa}),\left(\sigma_{\text {uts }}\right)$ & 463 & 452 & 432 & 414 & 466 & 450 \\
\hline
\end{tabular}

Fig. 4 shows magnified views of unloading followed by loading curves for two separate steps of the interrupted test on the sample aligned with $\mathrm{RD}\left(0^{\circ}\right)$. Fig. 4a illustrates the unloading-loading curve for loop 4 (unloading) to 5 (loading), where the difference between the initial elastic modulus (105GPa, before yield) and that during the unloading of the loop 4 is more evident. This is in line with expectations since the plastic deformation causes damage to accumulate in the microstructure and affects the elastic modulus. Once the sample was unloaded, the slope was initially linear (new elastic modulus) down to just below 100MPa, where the linear relaxation changed to nonlinear (i.e. 
change in the slope) and more recovery of strain was observed. This type of nonlinear recovery was observed for all the steps during the interrupted tensile tests. Fig. 4b shows a similar nonlinear recovery for loop 6 to loop 7 . This nonlinear recovery occurred below $100 \mathrm{MPa}$ in all cases. The red dotted lines in Fig. 4b shows the change in the slope following unloading clearly. A noticeable reduction was observed in the final strain from that expected based on the initial elastic strain. This reduction can be separated into two segments: A and B. Segment A is mainly the result of a reduction in the elastic modulus. Some studies $[5,7]$ have considered this degradation in the elastic modulus, however, most of them were focused on only one direction of the sheet. Since a noticeable difference in the elastic modulus of samples with different orientations is observed, it is necessary to study this degradation in all directions for the current material.

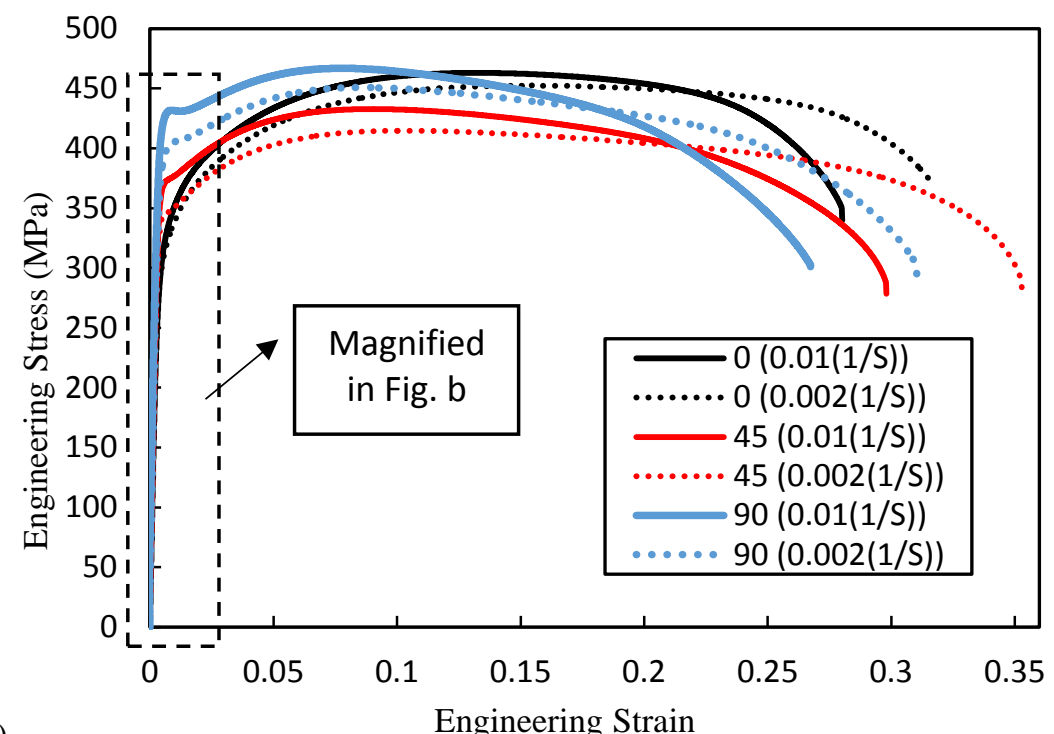

a)

Engineering Strain

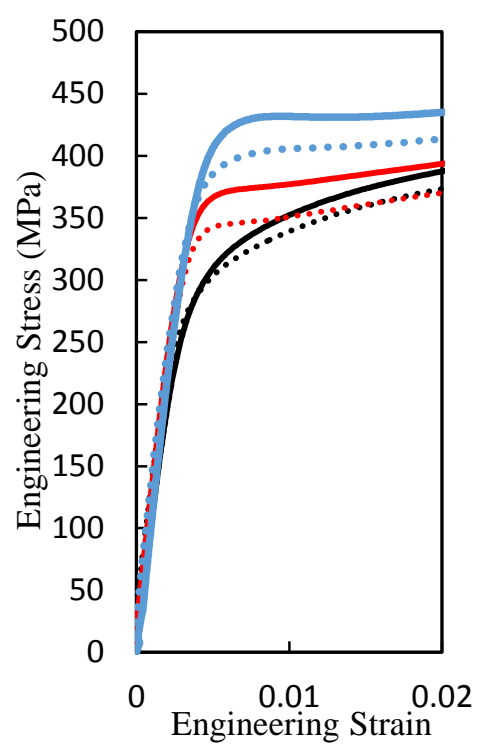

Fig. 2: Engineering stress-strain curves of CP-Ti samples orientated in different directions of $0^{\circ}, 45^{\circ}$ and $90^{\circ}$ to the $\mathrm{RD}$, tested with different strain rates: a) full tests, b) Magnified elastic region.

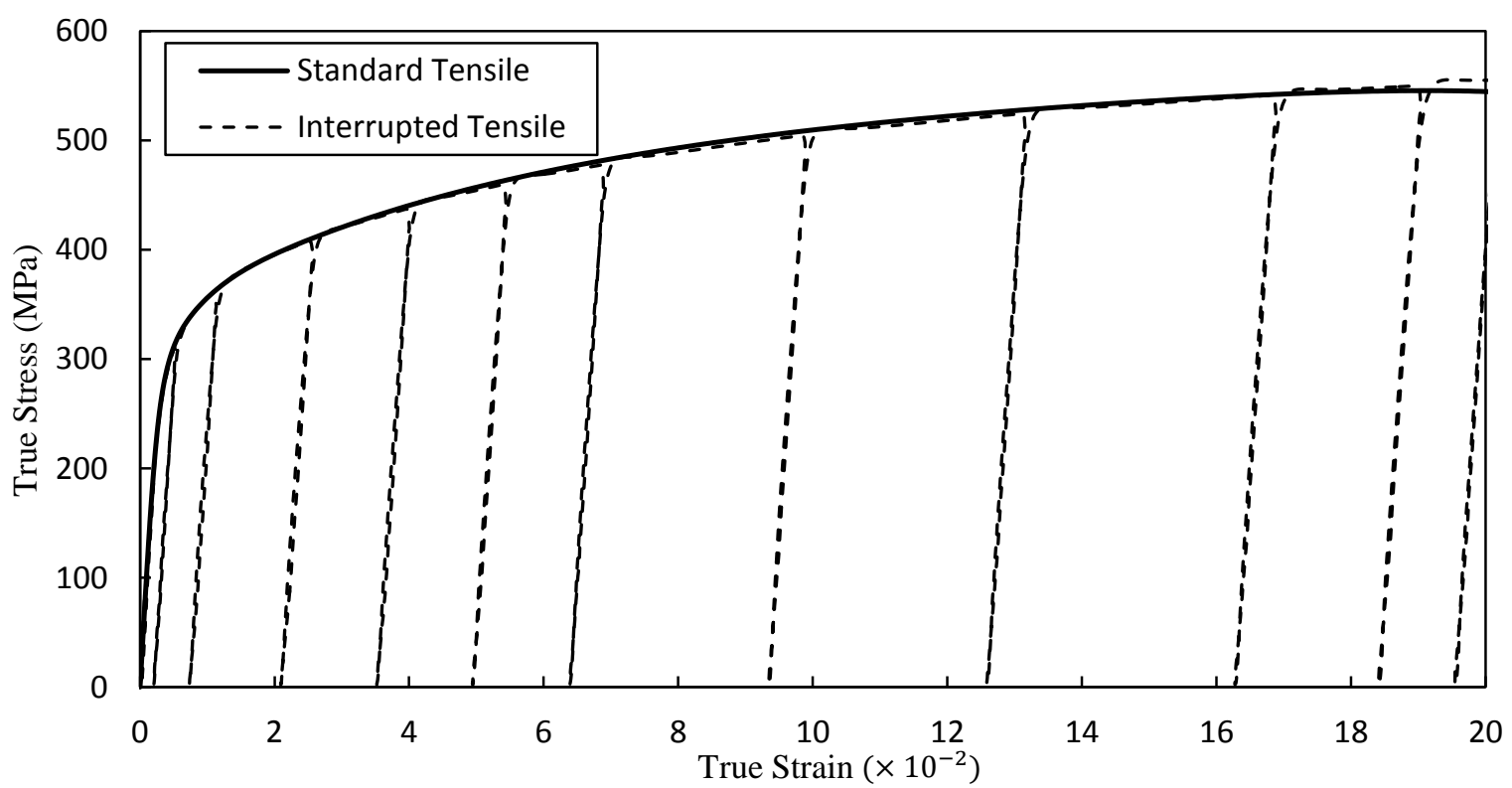

Fig. 3: True stress-strain curve for a continuous and an interrupted (loading-unloading) tests, up to UTS for the samples at $0^{\circ}$ (aligned with the RD), with strain rate of $0.01 \mathrm{~s}^{-1}$.

Segment B in Fig. $4 \mathrm{~b}$ is also an important reduction in the total strain which could be the microplastic recovery in the material. Neglecting segment B would result in inaccurate results for estimating springback, whereas, in most studies this nonlinear recovery (segment B) was not 
observed or has been neglected [4-7]. However, there is a possibility that this nonlinear recovery is dependent on the material characteristics such as dislocation motion behaviour and may not be similar in other materials. Therefore, estimating the non-linear recovery in different directions and considering it in a predictive model is crucial in controlling springback more accurately.
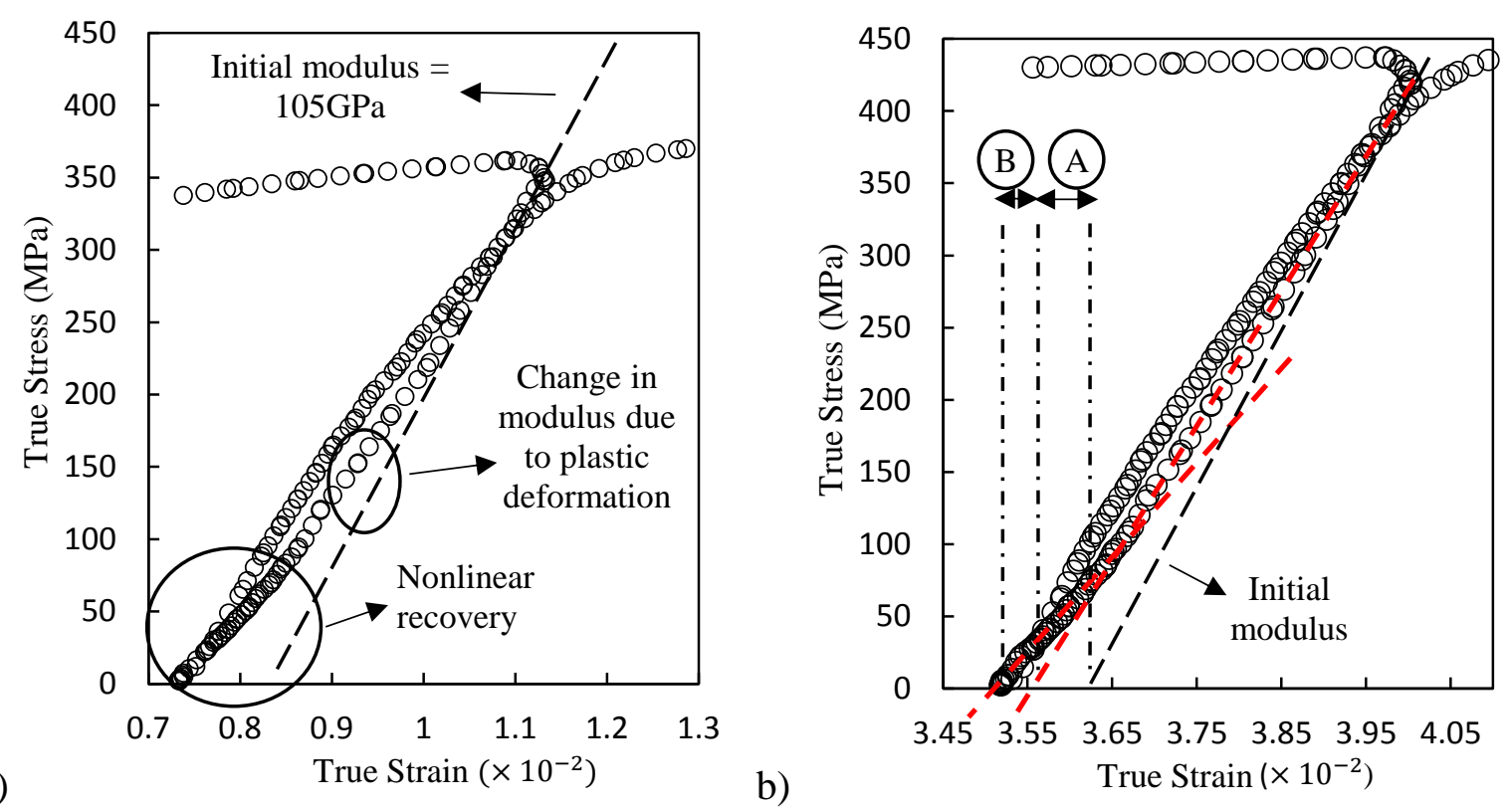

Fig. 4: Magnified views of unloading data for a sample at $0^{\circ}$ (i.e. aligned with RD), showing the nonlinear recovery following the unloading. a) unloading-loading curve for loop 4 to 5 . b) unloading-loading curve for loop 6 to 7.

Fig. 5a shows the degradation in elastic modulus as a function of true plastic strain for samples at $0^{\circ}$ and $45^{\circ}$ of RD. Despite the fact that elastic modulus is higher for the sample at $45^{\circ}$ degree compared to that of $0^{\circ}$, both showed a similar reduction in $E$, especially after 0.05 true plastic strain. Both directions exhibited a significant decrease in their elastic modulus, by $50 \%$ reduction after almost 0.25 true plastic strain. This result confirms that there is a substantial decline in elastic modulus by plastic deformation that it is necessary to account for in springback predictive models (Fig. 5a).

Fig. 5b shows the nonlinear (plastic) strain, identified as segment B in Fig. 4b, as a function of plastic strain introduced during the loading-unloading process for both directions of $0^{\circ}$ and $45^{\circ}$ to $\mathrm{RD}$. This nonlinear recovery, which is assumed to be part of the recovered plastic deformation, demonstrated a different behaviour, in terms of magnitude, in $0^{\circ}$ compared to that of $45^{\circ}$. For both directions, the initial values were zero, corresponding to the first two loading-unloading steps below the yield point, indicating that no plastic deformation had occurred. This value was then increased in each case once the onset of plastic deformation started. The magnitude of plastic recovery for samples at $0^{\circ}(\approx 0.0003$ true plastic strain $)$ is almost three times of that of the sample at $45^{\circ}$ $(\approx 0.0001$ true plastic strain). These results confirm that the magnitude of plastic recovery depends on the direction of the sample and more tests need to be performed in other directions to capture material behaviours to feed into a predictive model of springback.

\section{Discussion}

As shown, there is a significant reduction in the elastic modulus $(\approx 50 \%)$ as a function of plastic deformation. This is measured to be almost the same for samples in the two directions at $0^{\circ}$ and $45^{\circ}$ degrees to RD. An additional measureable plastic strain recovery is observed after each unloading step of interrupted (i.e. loading-unloading) tensile tests. This is contributing toward the total strain recovered after unloading and therefore causing significant springback during sheet metal forming. In order to predict springback more precisely, it is necessary to construct a predictive model which 
takes into account the degradation in elastic modulus as a function of plastic deformation, for each orientation of the sheet. The predictive model also needs to account for the extra plastic recovery, which can be significant at some directions, such as at $0^{\circ}$. It is important to conduct more research to study this nonlinear recovery, as this might not be crucial for all materials. The present work is part of ongoing research and the current recommendations and achievements will be taken into account.
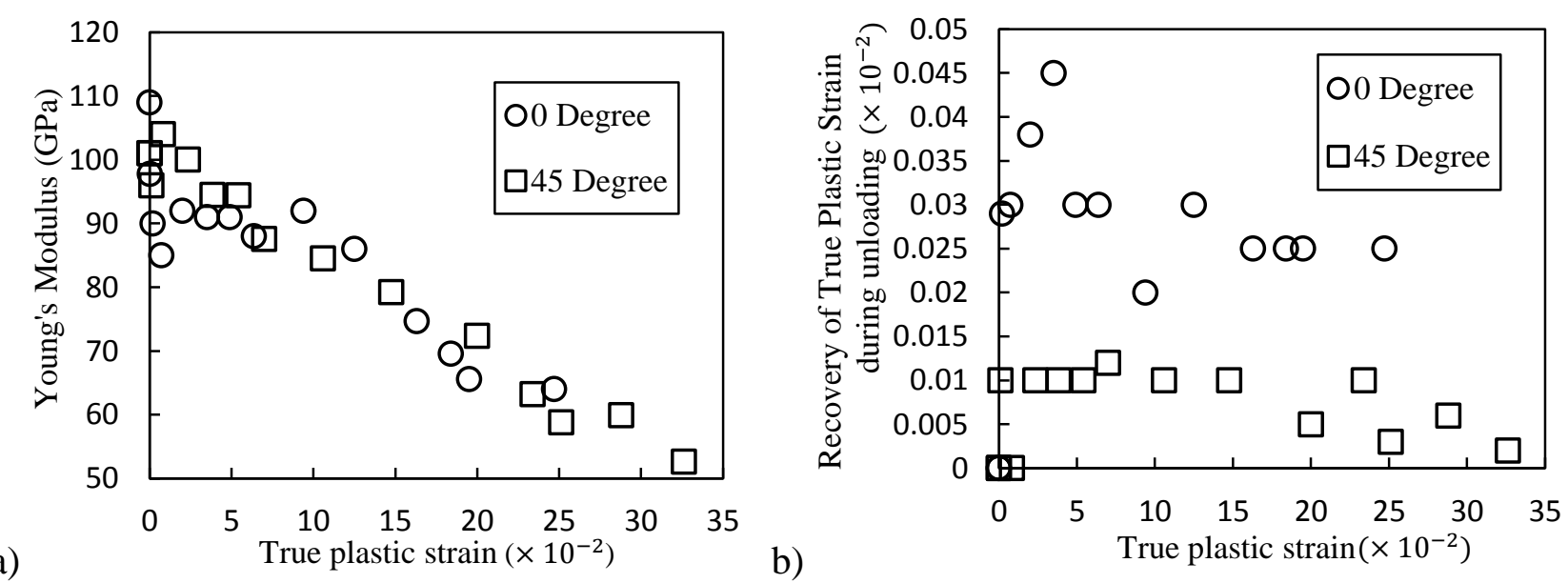

Fig. 5: a) Degradation of Young's modulus as a function of true plastic strain during the loadingunloading tests. b) Recovery of true plastic strain after each unloading step as a function of plastic strain induced during loading-unloading tests.

\section{Summary}

- It can be concluded that the mechanical properties of CP-Ti vary as a function of direction within the sheet.

- The interrupted tensile tests showed that the elastic modulus is reduced by up to $\approx 50 \%$, with an increase in the plastic deformation $(\approx 25 \%)$, during the loading-unloading. This was observed for tests carried out at $0^{\circ}$ and $45^{\circ}$ to RD.

- An extra nonlinear (plastic deformation) recovery was observed after each unloading step which could be notified by a change in the slope of unloading curve. The amount of plastic recovery for samples at $0^{\circ}$ of the RD was noticeably higher than those for samples at $45^{\circ}$.

\section{References}

[1] J.C. Eglin, O. Gouty, D. Joanna, Shape and process parameters optimization for the accurate control of springback in sheets metal forming, Sheet Metal, Vol. 2, Univ. of Twente, (1996) 3-14

[2] D. Joannic, Modelisation mecanique et simulation du retour elastique en emboutissage des toles minces et optimisation parametrique, Ph. D. Thesis, Université de Franche-Compté, Besançon, (1998)

[3] I.A. Burchitz, Improvement of Springback Prediction in Sheet Metal Forming, Univ. of Twente, Rotterdam, The Netherlands, (2008)

[4] L. Luo, A.K. Ghosh, Elastic and inelastic recovery after plastic deformation of DQSK steel sheet, J. Eng. Mater. Technol. 125(3) (2003) 237-246

[5] J.A. Benito, J.M. Manero, J. Jorba, A. Roca, Change of Young's modulus of cold-deformed pure iron in a tensile test, Metall. Mater. Trans. A.s 36(12) (2005) 3317-3324

[6] R.M. Cleveland, A.K. Ghosh, Inelastic effects on springback in metals. Int. J. Plast. 18 (2002) 769-785 
[7] R. Perez, J.A. Benito, J.M. Prado, Study of the inelastic response of TRIP steels after plastic deformation, ISIJ Int. 45(2005) 12 1925-1933

[8] ASTM E8/E8M- 11. Standard Test Methods for Tension Testing of Metallic Materials. (2012) 